\title{
Écrire pour transmettre des connaissances procédurales : le cas des documents techniques procéduraux
}

\section{Franck Ganier}

\section{OpenEdition}

Journals

Édition électronique

URL : http://journals.openedition.org/pratiques/3168

DOI : $10.4000 /$ pratiques.3168

ISSN : 2425-2042

Éditeur

Centre de recherche sur les médiations (CREM)

Référence électronique

Franck Ganier, «Écrire pour transmettre des connaissances procédurales : le cas des documents techniques procéduraux », Pratiques [En ligne], 171-172 | 2016, mis en ligne le 07 février 2017, consulté le 19 avril 2019. URL : http://journals.openedition.org/pratiques/3168; DOI : 10.4000/pratiques.3168

Ce document a été généré automatiquement le 19 avril 2019

(c) Tous droits réservés 


\title{
Écrire pour transmettre des connaissances procédurales : le cas des documents techniques procéduraux
}

\author{
Franck Ganier
}

\section{Introduction}

$1 \quad$ Les documents techniques procéduraux font partie de notre quotidien. Ils se présentent sous des aspects multiples (recettes de cuisine, plans de montage, aides en lignes, notices pharmaceutiques, guides d'utilisation) et servent des buts aussi variés que la fabrication, l'installation, l'utilisation ou encore la maintenance de matériels et de dispositifs divers. Une caractéristique majeure de ces documents, à savoir leur visée pragmatique, permet de les distinguer sans peine d'écrits non techniques comme les romans ou les recueils de poésie. On les distingue parfois avec plus de difficulté des écrits techniques non procéduraux comme les articles d'encyclopédies, les fiches produits ou encore certains documents légaux (garanties, avertissements). Pour Wright (1987), un certain nombre d'éléments permettent de différencier ces différents types de documents : certains indices visuels (dans les documents techniques, la disposition spatiale du texte et des images répond à des contraintes fortes), le style rédactionnel (l'usage de l'impératif ou de l'infinitif est fréquent dans les documents procéduraux), la dépendance au contexte d'utilisation (très forte dans le cas des documents procéduraux), ou encore les contraintes d'usage (qui peuvent déterminer le format du document). Le process de conception des documents lui-même constitue un facteur de différenciation.

Dans le cas du document procédural, le rédacteur technique obtient généralement des informations d'un expert du domaine, par exemple un ingénieur chargé de la conception du produit, qui possède une bonne représentation de l'usage, des possibilités offertes et des limites de ce produit. Il doit retranscrire ces informations sous une forme intelligible 
en vue de les transmettre à un utilisateur qui ne possède - la plupart du temps - aucune connaissance du produit qu'il souhaite utiliser, ou dont les connaissances ne sont que parcellaires. La rédaction du document doit donc être orientée par l'utilisation du produit, en gardant à l'esprit que la priorité de l'utilisateur est d'interagir avec celui-ci (la lecture du document ne constitue pour lui qu'une activité secondaire qui doit à la fois l'assister dans la réalisation de son activité principale tout en étant la plus transparente possible). Il s'agit donc de communiquer des procédures de façon efficace, en présentant l'information de sorte qu'elle corresponde aux besoins de l'utilisateur, tout en évitant de le surcharger sur le plan cognitif.

3 Malheureusement, les documents procéduraux échouent parfois à remplir cet objectif. Ganier et Barcenilla (2007) entrevoient des causes multiples à ces échecs, issues à la fois des caractéristiques de l'utilisateur, du document, du dispositif et du contexte d'utilisation. Pour Westendorp (2000), il s'agit d'un problème de communication complexe lié au fait que les produits sont eux-mêmes complexes (ils offrent une diversité de variantes), que le public cible peut être très diversifié (en termes d'âge, de culture, de niveau d'éducation, de relation à la technologie, etc.), ce phénomène étant accentué par la mondialisation des échanges, et que les spécialistes en charge de la conception de ces documents ne se trouvent que très rarement - voire jamais - au contact direct d'utilisateurs et n'en reçoivent généralement pas de feedback.

Depuis plus de quarante ans, des études sont réalisées en psychologie cognitive et en ergonomie pour tenter d'identifier les facteurs impliqués dans l'utilisation de tels documents et pour proposer des solutions permettant d'en améliorer la qualité et l'efficacité. Ces études sont centrées en majeure partie sur l'utilisation et la compréhension par le destinataire des informations présentées dans les documents et accordent peu de place au rôle joué par le rédacteur technique, à son fonctionnement cognitif, au processus de rédaction, ou encore aux contraintes et aux difficultés rencontrées dans l'élaboration de tels documents. L'objectif de cet article est de s'appuyer sur les apports des travaux réalisés en psychologie cognitive et en ergonomie pour aider les rédacteurs techniques à améliorer l'efficacité des documents procéduraux. Dans cette perspective, il s'articulera autour de quatre questions. Il s'agira d'abord de déterminer les sources de difficultés auxquelles se trouvent confrontés les rédacteurs techniques, puis de se centrer sur le traitement des documents procéduraux par les utilisateurs pour identifier les facteurs cognitifs et ergonomiques pertinents à prendre en compte pour la conception de ces documents. L'ensemble sera complété par une présentation des apports des recherches sur la conception et la rédaction de documents techniques, d'une part à travers une présentation des modèles du process de conception de documents techniques, et d'autre part à travers une présentation des compétences que doivent acquérir les rédacteurs techniques pour une communication efficace des procédures.

\section{Les sources de difficultés auxquelles se trouvent confrontés les rédacteurs techniques}

5 Pour concevoir des documents qui soient utilisables par le public cible, les rédacteurs techniques se trouvent confrontés à un certain nombre de problèmes à résoudre. Ils doivent tout d'abord déterminer un contenu approprié, correspondant sur le fond aux informations transmises par l'expert du domaine, et sur la forme aux besoins des utilisateurs. Pour ce faire, il s'agira donc de cerner les caractéristiques du public cible, en 
particulier leurs connaissances et leurs attentes, à la fois au sujet du produit qu'ils auront à utiliser mais aussi au sujet de la documentation. Il s'agira aussi d'amener l'expert à verbaliser ses connaissances, de transcrire des procédures parfois complexes, ou de rédiger sous forme de procédures des informations délivrées sous une forme succincte dans une fiche produit. Le tout devant être exprimé dans un style qui puisse être aisément compris du public cible. Sur le plan rédactionnel, le rédacteur technique devra donc déterminer l'ordre d'importance des informations à transmettre, le niveau de granularité de la description du produit et des actions à réaliser, la formulation et la terminologie à utiliser, etc. Il devra également choisir le format de présentation à utiliser pour transmettre ces informations (texte, image, tableau, arborescence, etc.) ainsi que des éléments typographiques appropriés pour signaler aux utilisateurs les différents statuts de l'information présentée dans le document (titres, sous-titres, instructions, avertissements, questions/réponses, etc.). Certains choix peuvent être liés à des critères purement économiques. Par exemple, le besoin grandissant d'instructions multilingues se traduit par une utilisation accrue d'images (à l'instar des consignes de sécurité proposées par l'aviation commerciale).

6 Enfin, il devra déterminer le type d'organisation à adopter dans la mise en page. Par exemple, il s'agira d'articuler les éléments textuels et graphiques pour aider l'utilisateur à parcourir le document afin de localiser les informations pertinentes ou bien de lui faciliter la compréhension de la procédure. Ces décisions sont souvent prises avec une connaissance minimale et, la plupart du temps, purement intuitive de leurs effets sur l'utilisation des documents et sur la compréhension de l'information présentée. En effet, les rédacteurs techniques ne disposent que très rarement d'éléments les renseignant sur la façon dont les documents procéduraux sont utilisés et sur la façon dont les informations contenues dans ces documents sont traitées, comprises et appliquées (Ganier, 2006, 2013 ; Westendorp, 1997, 2000 ; Wright, 1981a). En effet, par opposition à une situation usuelle d'interlocution où les interlocuteurs disposent de feedbacks leur permettant de réajuster leur discours, la situation de production de documents procéduraux ne permet pas au rédacteur de bénéficier d'un feedback immédiat d'utilisateurs (Fayol, 2002), du fait de la rareté des contacts directs avec ceux-ci. Les utilisateurs n'interviennent que très rarement dans le processus de conception des documents techniques (Ganier, 2002a ; Valentin \& Lucongsang, 1987). Les seules informations susceptibles de renseigner le rédacteur sur la manière dont le document est traité sont accessibles en différé, par l'intermédiaire des retours-clients aux Services Consommateurs, Qualité, Après-Vente, etc. Dans une telle situation, les rédacteurs techniques font donc appel à un modèle implicite plus ou moins précis des utilisateurs et de leurs connaissances. En conséquence, il leur est très difficile - voire impossible - de repérer leurs éventuelles difficultés et sources d'incompréhension. D'autant qu'il existe une grande variété d'utilisateurs, présentant des caractéristiques différentes en termes de capacités de lecture, de connaissances concernant le produit à manipuler, ou encore de familiarité avec la tâche à réaliser. Au niveau de la rédaction du document procédural, Ganier et Barcenilla (2007) constatent que cette distance entre le rédacteur et l'utilisateur peut se traduire par une mise en forme inadéquate. Dans ce cas, les aspects typographiques et dispositionnels du texte ne permettent pas de percevoir le caractère hiérarchique des informations, et ne facilitent ni les nécessaires prises d'information sur le texte lors des allers-retours entre le document et le dispositif, ni la mémorisation des instructions. Elle peut également se traduire par un nombre important d'informations 
non mentionnées car implicites chez le rédacteur (ou chez l'expert du domaine), par un ordonnancement incorrect des instructions, par des écarts entre la terminologie utilisée par les rédacteurs et celle des utilisateurs ou par un niveau d'abstraction des instructions trop élevé, conduisant à un guidage insuffisant, ou au contraire, un niveau de granularité trop fin, avec pour conséquence un risque de "surcharge" d'information.

Conscients de ces difficultés, certains industriels ont mis en place des comités de rédaction constitués d'experts internes à l'entreprise (par exemple, responsables des services Marketing, Qualité, Après-Vente, Laboratoire d'essais, etc.). Ceux-ci sont chargés de relire et réviser le document avant sa diffusion (Ganier 2002a ; Valentin \& Lucongsang, 1987). Toutefois, du fait de leur niveau élevé de connaissance du dispositif et des opérations possibles sur celui-ci, et d'une méconnaissance de ses utilisateurs, il semblerait que ces experts éprouvent des difficultés pour repérer ou se représenter les éventuels problèmes de compréhension du document et d'utilisation du dispositif (Ganier, Gombert, \& Fayol, 2000 ; Heurley \& Ganier, 2002 ; Kern, 1985 ; Veyrac, Cellier, \& Bertrand, 1997). Par ailleurs, il arrive bien souvent que les membres des comités de rédaction effectuent les révisions en l'absence de manipulation du dispositif (par exemple, parce qu'il n'existe que deux ou trois prototypes fonctionnels dans l'entreprise, et qu'aucun n'est disponible au moment de l'évaluation du document). Dans ce cas, ils seraient amenés à se centrer sur l'évaluation et la correction des aspects de surface plutôt que des aspects pragmatiques des informations contenues dans le document (Ganier \& Pétillon, 2011).

8 Une part des difficultés rencontrées par les rédacteurs techniques peut provenir d'un manque de formation : Flacke, en 2005, estimait de 80000 à 100000 le nombre de rédacteurs techniques en France alors que le nombre de formations de niveau Master conduisant à ce métier était estimé à moins d'une dizaine. Ces constats rejoignent ceux de Vanhulle (2000) et Clerc et Beaudet (2002) qui soulignent le manque de formations institutionnalisées dans le domaine de la rédaction technique dans les pays de l'Europe francophone. Les rédacteurs techniques se trouveraient donc contraints d'acquérir des compétences en situation d'autoformation. Le risque est alors d'appliquer des règles de rédaction, du type de celles fréquemment rencontrées dans des manuels de rédaction technique anglo-saxons, sans véritablement en connaître les effets sur le comportement de l'utilisateur. Ainsi, lorsqu'ils se limitent à cette approche, les rédacteurs techniques ne bénéficient a priori d'aucun élément susceptible de les renseigner sur l'efficacité de leurs choix de conception (ou de rédaction), alors qu'ils pourraient s'appuyer sur les résultats des recherches qui s'emploient à mieux connaître le fonctionnement cognitif de l'utilisateur et à déterminer les effets de la conception d'instructions sur celui-ci. Malheureusement, ces recherches souffrent d'un manque de transfert des connaissances acquises vers les rédacteurs techniques. C'est un aperçu de ces travaux qui sera présenté dans ce qui suit.

\section{Les apports de la psychologie cognitive ergonomique à la conception d'instructions}

Dès 1965, Chapanis attirait l'attention des chercheurs sur un domaine négligé jusque-là : le langage associé aux outils, machines, systèmes et à leur utilisation. Il s'appuyait sur un certain nombre d'exemples concrets d'instructions pour rendre compte des difficultés de compréhension des utilisateurs et proposer des améliorations de ces instructions. Cet 
appel a été repris par Broadbent (1977), qui se basait sur des exemples tirés de la vie courante pour déduire des principes de rédaction (utiliser des instructions simples et courtes, éviter la forme passive, etc.). Pour dépasser cette approche, Broadbent suggérait que des expérimentations systématiques portant sur la compréhension du langage pouvaient venir en appui à l'ergonomie dans ce domaine. Ce type de travaux était en effet resté anecdotique jusqu'au milieu des années 1970, époque à laquelle s'est opérée une convergence entre des travaux issus majoritairement de la psychologie cognitive (Jones, $1966,1968)$ et des travaux issus de l'ergonomie (Sremec, 1972). Cette nouvelle approche du "document design" ou "ergonomie de documents" s'est notamment cristallisée dans les travaux de Patricia Wright ${ }^{1}$ au Royaume-Uni, qui visait à étudier l'ensemble des facteurs susceptibles d'améliorer la conception et l'utilisation des documents procéduraux.

Depuis cette époque, des travaux sont donc conduits en psychologie cognitive et en ergonomie dans les domaines de la production, de la compréhension et de l'évaluation des documents procéduraux. Les études réalisées montrent généralement que des variations dans la présentation des instructions (que ce soit au niveau de la terminologie, de la syntaxe ou du format de présentation de l'information) affectent le comportement de l'utilisateur (sa compréhension, ses performances, etc.). Au-delà de ce type de constat et des préconisations de conception de documents qui en découlent, ces travaux ont naturellement permis d'élaborer de nouvelles méthodes et de nouveaux outils d'investigation et d'évaluation des documents procéduraux et ont débouché sur l'élaboration de modèles de fonctionnement de l'utilisateur (pour une revue des principaux modèles, voir Heurley \& Ganier, 2006 et Ganier, 2012). Ces modèles peuvent servir de cadre de référence pour le rédacteur technique en lui permettant par exemple de mieux comprendre le rôle joué par les différentes sources d'information sur les processus cognitifs en jeu.

11 Outre les aspects méthodologiques et les apports liés à une meilleure connaissance du fonctionnement de l'utilisateur, les résultats qui découlent des travaux réalisés peuvent aider le rédacteur technique à prendre des décisions concernant la meilleure façon de présenter l'information dans un document. En effet, ces travaux permettent d'identifier les facteurs susceptibles de faciliter ou de perturber les traitements, facteurs qu'il est nécessaire de prendre en compte pour concevoir des documents adaptés. Ainsi, l'étude des relations entre le type de tâche à réaliser (jeu, assemblage, maintenance, etc.), le profil des utilisateurs (individus jeunes ou âgés, novices ou experts du domaine, etc.), le caractère immédiat ou différé de l'exécution de la procédure et le format de présentation des instructions (texte, image, vidéo, etc.) devrait permettre de guider les rédacteurs dans le choix de l'utilisation de l'un ou l'autre des formats de présentation d'information. Par exemple, Mykityshyn, Fisk et Rogers (2002) ont étudié l'effet d'utilisation de vidéo sur la manipulation d'un appareil de mesure de la glycémie, comparativement à l'utilisation d'un mode d'emploi classique. Ils ont mis en évidence que les différents formats de présentation n'affectaient ni la performance de calibrage de l'appareil par les jeunes adultes, ni sa durée. En revanche, les adultes âgés réussissaient mieux et plus rapidement la tâche lorsque les instructions étaient présentées sous forme de vidéo lors de la prise en main de l'appareil. Ces résultats vont naturellement en faveur de l'usage d'instructions vidéo pour la formation des individus âgés à l'utilisation de ce genre d'appareil. De tels exemples de recherches peuvent se décliner à l'envi. Toutefois, Westendorp (1997) considère que le focus des recherches est inapproprié car il est encore trop centré sur des détails de conception des documents. Pour Fayol (2002), il s'agit de "micro-recherches" 
qui restent parcellaires et isolées, élaborées la plupart du temps en réponse à des questions immédiates. Pour ces auteurs, il s'avère nécessaire d'élargir les recherches à des aspects plus globaux de la conception des documents. Cette perspective est abordée dans la section suivante.

\section{Les modèles du process de conception de documents techniques}

En 1980, Hayes et Flower ont proposé un modèle de production de texte articulé autour de trois types de processus rédactionnels : planification, mise en texte et révision. Dans le domaine de la rédaction technique, un certain nombre d'auteurs ont proposé des modèles de conception de documents procéduraux apparentés à ce modèle ${ }^{2}$. Ces modèles sont représentés de manière synthétique dans le Tableau 1. Ainsi, Felker (1980, cité par Wright, 1981b, c) a présenté un modèle de process de conception en trois étapes. La première étape, dite "pré-conception", consiste à déterminer quels sont les besoins d'information de l'utilisateur et sous quelle forme cette information doit être présentée. Pour Felker (1980), les besoins des utilisateurs peuvent être recueillis à l'aide d'entretiens ou d'enquêtes par questionnaires. Lorsque le contenu des instructions a été ainsi déterminé, il s'agit de définir sous quelle forme l'information doit être présentée : par exemple, il peut s'agir de choisir entre du texte et des images. Bien entendu, cette étape passe par une phase d'identification du public cible, mais aussi des contraintes contextuelles imposées à la fois par le dispositif, par la façon dont sera diffusé le document et par la façon dont il sera utilisé. Lorsque ces choix ont été opérés, la deuxième étape, ou "étape de conception", se traduit par la rédaction d'une première version (un brouillon ou draft) qui intègre les éléments recueillis lors de l'étape précédente : il s'agit d'organiser l'information et de rédiger dans un style clair pour les utilisateurs. Ces deux étapes sont complétées par une étape de "post-conception". Ici, il s'agit pour le rédacteur de relire et d'évaluer le brouillon en vue de déterminer que le document atteint bien son objectif. Bien que cette étape n'implique pas la participation du public cible, pour Felker (1980) la relecture et l'évaluation du document par le rédacteur auront nécessairement des répercussions sur sa révision.

Tableau 1. Modèles du process de conception de documents techniques

\begin{tabular}{|l|l|l|l|l|}
\hline Etape & $\begin{array}{l}\text { Felker (1980) } \\
3 \text { étapes }\end{array}$ & $\begin{array}{l}\text { Sullivan } \\
\text { Chapanis (1983) } \\
\text { étapes }\end{array}$ & $\begin{array}{l}\text { Lopinto (1984) } \\
8 \text { étapes }\end{array}$ & $\begin{array}{l}\text { Chapanis (1988) } \\
10 \text { étapes }\end{array}$ \\
\hline
\end{tabular}




\begin{tabular}{|c|c|c|c|c|}
\hline 1 & $\begin{array}{l}\text { Pré-conception. } \\
\text { Identification des } \\
\text { besoins } \\
\text { d'information de } \\
\text { l'utilisateur. } \\
\text { Prises de décision } \\
\text { concernant la forme } \\
\text { sous laquelle } \\
\text { l'information doit } \\
\text { être présentée. }\end{array}$ & $\begin{array}{l}\text { Revue de } \\
\text { littérature sur la } \\
\text { rédaction } \\
\text { d'instructions. } \\
\text { Identification d'un } \\
\text { certain nombre de } \\
\text { règles de rédaction. }\end{array}$ & $\begin{array}{lll}\text { Conception } & \text { de } & \text { la } \\
\text { structure } & & \text { du } \\
\text { document. } & & \\
& & \end{array}$ & $\begin{array}{l}\text { Elaboration d'un } \\
\text { scénario d'usage du } \\
\text { dispositif. }\end{array}$ \\
\hline 2 & $\begin{array}{l}\text { Conception. } \\
\text { Rédaction d'une } \\
\text { première version } \\
\text { (organisation de } \\
\text { l'information et } \\
\text { rédaction dans un } \\
\text { style clair). }\end{array}$ & $\begin{array}{l}\text { Analyse des } \\
\text { tâches pouvant } \\
\text { être réalisées avec } \\
\text { le dispositif. }\end{array}$ & $\begin{array}{ll}\text { Collecte } & \text { et } \\
\text { organisation } & \text { de } \\
\text { l'information. } & \end{array}$ & \begin{tabular}{|lr} 
Recensement & des \\
contraintes & \\
auxquelles & doit \\
répondre & le \\
document. &
\end{tabular} \\
\hline 3 & $\begin{array}{l}\text { Post-conception. } \\
\text { Relecture et } \\
\text { évaluation de la } \\
\text { première version } \\
\text { pour vérifier que le } \\
\text { document atteint } \\
\text { bien son objectif. }\end{array}$ & $\begin{array}{l}\text { Rédaction d'une } \\
\text { première version ( } \\
\text { draft). }\end{array}$ & $\begin{array}{l}\text { Rédaction d'un } \\
\text { plan détaillé. }\end{array}$ & $\begin{array}{l}\text { Analyse } \\
\text { documentaire } 1 \text { : } \\
\text { étude de documents } \\
\text { similaires à celui qui } \\
\text { doit être conçu. }\end{array}$ \\
\hline 4 & & $\begin{array}{lr}\text { Test de } & \text { mise } \\
\text { oeuvre } & \text { en } \\
\text { walkthrough } & \text { test). } \\
\text { Lecture r } & \text { du } \\
\text { document } & \text { étape } \\
\text { par étape } & \text { en } \\
\text { exécutant } & \text { les } \\
\text { actions décrites. }\end{array}$ & $\begin{array}{l}\text { Rédaction d'une } \\
\text { première version } \\
(d r a f t) \text {. }\end{array}$ & $\begin{array}{lll}\text { Analyse } & & \\
\text { documentaire } & \mathbf{2} \\
\text { consultation de } & \text { la } \\
\text { littérature } & & \\
\text { concernant } & & \text { la } \\
\text { conception } & \text { de } \\
\text { documents. } & & \end{array}$ \\
\hline 5 & & $\begin{array}{l}\text { Inspection } \\
\text { d'utilisateurs ( } \\
\text { user } \text { edit). } \\
\text { Un échantillon } \\
\text { d'utilisateurs } \\
\text { réalise les tâches } \\
\text { avec l'objet en } \\
\text { suivant les } \\
\text { instructions. }\end{array}$ & $\begin{array}{l}\text { Vérification de la } \\
\text { précision technique } \\
\text { du contenu. }\end{array}$ & $\begin{array}{l}\text { Identification des } \\
\text { utilisateurs auxquels } \\
\text { le document est } \\
\text { destiné. }\end{array}$ \\
\hline
\end{tabular}




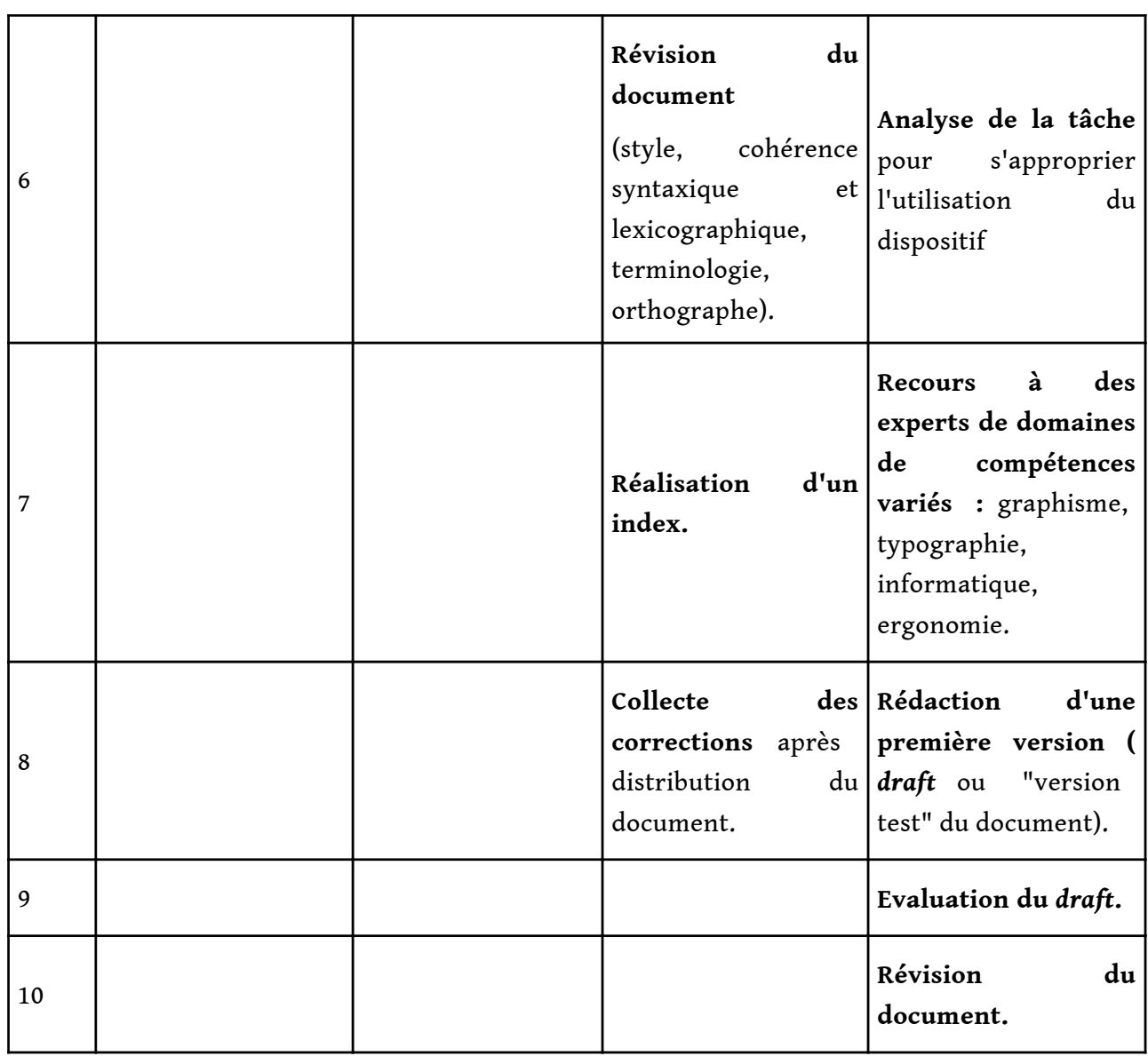

13 Une approche sensiblement différente a été envisagée par Sullivan et Chapanis (1983). À partir de leur propre expérience de rédaction du manuel d'un logiciel de traitement de texte, ces auteurs proposent une méthode de conception de la documentation technique en cinq étapes. La première étape s'appuie sur une revue de la littérature portant sur la rédaction d'instructions. Il s'agit ici d'identifier un certain nombre de règles susceptibles de guider la rédaction du document (utiliser un langage simple ; rédiger des phrases courtes à la forme active; présenter les instructions dans le même ordre que les actions ; décrire une seule action à la fois ; utiliser des titres et des soustitres pour identifier les différentes sections des instructions ; utiliser des listes plutôt que de longs paragraphes, etc.). La deuxième étape consiste à analyser les tâches qui peuvent être réalisées avec le dispositif. L'analyse de la tâche permet d'identifier les informations qui doivent figurer dans le document. Ces informations concernent tout autant les séquences d'opérations "normales" que les séquences "anormales", nécessitant des avertissements ou des mises en garde. La troisième étape consiste à rédiger une première version du document (draft) à partir des règles issues de la revue de littérature et de l'analyse des tâches. Ce draft est alors utilisé pour l'étape suivante, qui consiste à lire le document étape par étape et à exécuter les actions décrites. Ce test de mise en oeuvre ( walkthrough test) conduit à identifier les étapes manquantes et à suggérer des modifications qui peuvent être intégrées à une nouvelle version. Enfin, la cinquième et dernière étape, nommée "inspection d'utilisateur" (user edit), consiste à demander à un échantillon d'utilisateurs issus du public cible de réaliser les tâches avec l'objet en suivant les instructions. En notant la nature des problèmes rencontrés et leur fréquence d'occurrence, il est possible de réviser le texte. 
14 S'appuyant sur son expérience de terrain, Lopinto (1984) propose une procédure de conception / rédaction en huit étapes. La première étape porte sur la conception de la structure du document. Ici, il s'agit de déterminer de façon schématique quelles informations seront à inclure dans le document. Celui-ci peut être conçu sous forme de modules présentant systématiquement les informations procédurales liées à la manipulation de l'équipement, la récupération d'incidents et la maintenance (voir par exemple Ganier, 2002b, sur les aspects de conception modulaire). La deuxième étape consiste à collecter et organiser l'information. Lorsque la structure du document a été déterminée, il s'agit de collecter l'information et de la répartir sous les différents intitulés. Vient ensuite une étape de rédaction d'un plan détaillé. Cette troisième étape consiste à élaborer une liste de sous-titres pour chacun des titres ou modules principaux. Ces sous-titres, avec l'information qui leur est rattachée, devront être organisés sous forme de séquences logiques. Lorsque le matériel a été collecté et organisé, la rédaction d'un draft peut alors commencer, puisque les trois étapes précédentes auront permis au rédacteur (ou à l'équipe de rédaction) de se familiariser avec les détails techniques. La cinquième étape consiste à vérifier la précision technique. Cette étape se déroule en collaboration avec les ingénieurs responsables du projet qui devront réviser chaque section de cette première version du document. Pour Lopinto (1984), l'accent doit être mis sur le fait qu'il s'agit de vérifier la précision technique et non le style du document. Ce n'est que lorsque la précision technique du document a été vérifiée que le document peut être révisé par le rédacteur technique. Au cours de cette sixième étape, il s'agit d'examiner le style, la cohérence syntaxique et lexicographique, la terminologie utilisée et l'orthographe. Lopinto recommande ensuite de réaliser un index. Celui-ci devrait être utile aux utilisateurs familiers avec l'équipement ou la procédure qui souhaiteraient retrouver une information particulière. Enfin, la dernière étape intervient suite à la diffusion du document. Pour l'auteur, il est important de mettre en place un système permettant de recueillir des corrections après la distribution du manuel, dans le but de les intégrer à une nouvelle version.

Chapanis (1988) a repris et enrichi le modèle qu'il avait proposé avec Sullivan en 1983 pour en proposer une version en dix étapes. La première étape consiste à élaborer un scénario d'usage. Il s'agit ici de décrire la façon dont le produit sera utilisé, à quel endroit, sous quelles conditions et pour quoi faire. Ce scénario devrait permettre d'aider le rédacteur à recenser - dans une deuxième étape - les contraintes auxquelles doit répondre le document. Par exemple, il devra tenir dans la poche du technicien de maintenance ; de nouvelles pages devront pouvoir y être ajoutées régulièrement; il devra être compris d'une personne de tel niveau d'étude ; etc. Les troisième et quatrième étapes portent sur l'analyse de documents. D'une part, il s'agit d'étudier des documents similaires à celui qui doit être conçu. D'autre part, il s'agit de consulter la littérature concernant la conception de documents. Pour Chapanis (1988), ces étapes d'analyse documentaire préliminaires à la rédaction peuvent s'avérer fructueuses et seront toujours utiles pour les étapes suivantes. La cinquième étape consiste à identifier les utilisateurs. Pour Chapanis (1988), il est en effet tout aussi important de connaître le profil des personnes à qui un document est destiné que de connaitre le profil des personnes à qui un produit est destiné. La sixième étape proposée par Chapanis (1988) consiste à réaliser une analyse de la tâche dans le but de s'approprier l'utilisation du dispositif. Pour l'auteur, cette analyse de la tâche devrait permettre au rédacteur de se représenter ce que devra faire l'utilisateur lorsqu'il sera confronté au produit. La 
septième étape consiste à faire appel à des spécialistes de domaines dans lesquels le rédacteur technique n'est pas compétent, par exemple, les domaines du graphisme, de la typographie, de l'informatique ou encore de l'ergonomie. Lorsque tous ces éléments ont été rassemblés, le rédacteur peut alors rédiger un draft, que Chapanis (1988) considère comme une version test du document. La neuvième étape consiste à évaluer cette première version du document (pour la présentation de différentes méthodes d'évaluation des documents procéduraux, voir par exemple Ganier, 2002a ; Schriver, 1989 ; Wright, 1988a). Sur la base des résultats de l'évaluation, il s'agira de réviser le document lors de la dixième étape. Un certain nombre d'améliorations du document peuvent être opérées pour en améliorer l'efficacité et la qualité. Pour Chapanis, cette étape ne constitue pas l'ultime étape de conception, puisque les étapes 9 et 10 peuvent être répétées autant que nécessaire. L'auteur insiste sur le fait qu'il n'estime pas réaliste d'avoir pour objectif de créer un document parfait, c'est-à-dire un document avec lequel aucun utilisateur ne rencontrera des difficultés d'utilisation. La décision de mettre fin à ce processus itératif va donc dépendre du nombre d'utilisateurs qui rencontrent encore des difficultés, la nature de ces difficultés, le coût de ces difficultés pour les utilisateurs et la facilité avec laquelle elles peuvent être éliminées. Ce modèle constitue sans doute le modèle du process de conception de documents techniques le plus abouti à ce jour.

Dans la mesure où peu de recherches se sont intéressées au process de conception des documents techniques depuis les années 1980, on peut regretter l'absence de modèles plus récents. Toutefois, dans la mesure où l'étude de la rédaction connaît un regain d'intérêt depuis quelques années (en atteste l'ouvrage de Torrance et al., 2012) il ne fait aucun doute que l'étude du process de conception de documents techniques devrait bénéficier de ce nouvel élan. L'analyse des modèles présentés plus haut permet d'aboutir à deux remarques. D'une part, une comparaison de ces modèles (Tableau 1) permet d'extraire un certain nombre d'éléments nécessaires à prendre en compte dans la conception d'un document technique. Ainsi, l'identification de l'utilisateur, de ses besoins et des possibilités offertes par le produit; la connaissance de règles de rédaction; l'élaboration d'une première version du document; l'évaluation de la première version; l'implication d'utilisateurs dans l'évaluation et enfin, la révision du document apparaissent comme des étapes récurrentes dans la plupart des modèles. D'autre part, et dans la continuité de la remarque précédente, ces modèles ont un rôle indéniable à jouer dans le futur de la rédaction technique. En effet, ils peuvent constituer des ressources propres à l'enseignement de la rédaction technique et à la formation des futurs rédacteurs techniques, par exemple en étant utilisés comme base d'un enseignement méthodologique à intégrer dans les cursus de formation.

On le voit dans ces modèles, le process de conception implique la coordination d'un certain nombre de compétences des rédacteurs techniques en termes de communication écrite et graphique, en termes de familiarité avec la littérature développée par la recherche et en termes de techniques d'évaluation des documents. L'identification de ces compétences fera l'objet de la partie suivante.

\section{Les compétences des rédacteurs techniques}

La multiplicité et la diversité des étapes du process de conception de documents techniques énumérées plus haut suggèrent que la rédaction technique s'étend bien audelà d'une simple activité d'écriture, et requiert des compétences dans différents 
domaines (Clerc et Beaudet, 2002). L'identification de ces compétences constitue un enjeu de taille, car elle peut permettre de déterminer les programmes de formation à développer en direction des rédacteurs techniques (voir par exemple Clerc \& Beaudet, 2002, à ce sujet). Wright (1981b, c ; 1987) a entamé un travail de recensement des différents types de compétences dont doivent faire preuve les rédacteurs techniques. Pour cet auteur, cinq types de compétences sont nécessaires pour la conception de documents techniques. Ces savoir-faire concernent :

- L'analyse de la tâche. Ici, il s'agit de déterminer a priori quelles seront les conditions d'utilisation du document. Savoir comment un document sera utilisé peut s'avérer très utile pour déterminer son format ou encore le support sur lequel il sera présenté. Par exemple, si ce document doit être manipulé par un technicien de maintenance, à proximité immédiate de l'appareil, dans un espace restreint, dans la pénombre et qu'il doit être transporté, il devra respecter un format compatible avec l'ensemble de ces contraintes (voir Zafiharimalala, 2011).

- La maîtrise du langage, notamment pour ce qui concerne la rédaction de procédures complexes et la définition de termes techniques.

- La maîtrise de facteurs graphiques et typographiques. Le rédacteur technique doit savoir utiliser judicieusement des formats de présentation alternatifs au texte (graphiques, tableaux, etc.) et des options typographiques qui aident l'utilisateur à comprendre la structure sous-jacente du texte.

- L'analyse de travaux de recherche liés au domaine de la rédaction technique. Le rédacteur technique peut s'appuyer sur des travaux de recherche pour enrichir ses connaissances sur l'utilisation, la compréhension ou l'évaluation des documents techniques.

- La maitrise de l'ensemble du process de conception des documents : du recueil d'information auprès d'experts à la mise en production et la diffusion du document.

Plus récemment, Clerc \& Beaudet (2002) et Coppola (2011) ont déterminé un certain nombre de compétences de base (core competencies), au cœur du métier de rédacteur technique. Ainsi, Clerc \& Beaudet (2002) se sont notamment appuyées sur les travaux de Russell, Bossé-Andrieu \& Cajolet-Laganière (1995) pour établir un profil de connaissances, de compétences, mais aussi de qualités personnelles caractéristiques du rédacteur technique. Pour ces auteurs, le rédacteur technique n'est pas un expert en contenu, mais se situe d'abord comme un spécialiste de la communication écrite. Il doit notamment faire preuve d'une maîtrise parfaite de la langue et du discours, d'une bonne capacité de travail en équipe, d'adaptabilité, de rapidité d'exécution, tout en sachant répondre à des exigences de qualité. À cela s'ajoute un intérêt marqué pour la connaissance scientifique et technique. Pour Coppola (2011), le praticien confirmé doit faire preuve de compétences dans cinq domaines : l'analyse de l'utilisateur et de la tâche, la conception de l'information (Information design), la gestion du process de conception, le développement du contenu (à travers les processus de création et de révision du document) et la livraison $\mathrm{du}$ produit fini (comprenant sa publication, sa distribution et son archivage). Sur la base de ces travaux, des travaux de Wright (1981b, c, 1987) et des modèles de process de conception de documents techniques présentés plus haut, il est possible de redéfinir cinq classes de compétences :

- Des compétences méthodologiques. Ces compétences sont nécessaires à la fois pour recueillir des informations techniques et extraire les connaissances des experts du domaine en vue de rendre les procédures explicites, pour identifier les besoins des utilisateurs et pour évaluer l'efficacité des documents (sur ce dernier point, voir Ganier, 2002a). Les 
méthodes utilisées peuvent être aussi variées que la conduite d'enquêtes et d'entretiens, l'analyse de l'activité et la méthode expérimentale.

- Des compétences rédactionnelles et graphiques ou audiovisuelles. La maîtrise du langage écrit constitue bien évidemment une compétence de base - voire même le cœur de métier $\mathrm{du}$ rédacteur technique, aussi bien pour la rédaction que pour la révision du document. Toutefois, souvent, les rédacteurs techniques accordent davantage d'importance à la production et à la révision de texte, sans forcément porter attention à des modes alternatifs de présentation de l'information. Dans la mesure où les documents deviennent majoritairement multimodaux, notamment dans le cas de supports numériques, la compétence rédactionnelle n'est plus suffisante. Elle doit être associée notamment à des compétences graphiques ou audiovisuelles.

- Des compétences en recherche documentaire et analyse de l'information. Le rédacteur technique doit réaliser à la fois des activités de veille documentaire pour entretenir non seulement sa connaissance du domaine (analyse d'articles scientifiques liés à la rédaction technique), mais aussi sa connaissance de l'évolution du métier, des outils, etc.

- Des compétences liées aux usages du numérique. Le document procédural est encore très répandu au format papier, mais cède peu à peu la place au document numérique, pour des raisons multiples (Ganier, 2008, 2011 ; Toscano, 2000 ; Zafiharimalala, 2011). Par ailleurs, l'utilisation d'authoring tools, d'outils d'aide informatisée à la conception de documents techniques ou d'outils qui permettent de convertir des documents pour les adapter à différents supports (ordinateurs, tablettes, smartphones) fait partie du quotidien du rédacteur technique qui doit gérer une grande quantité d'information, souvent multilingue, qui nécessite des mises à jour fréquentes, etc. Enfin, l'évolution des systèmes techniques dessine des liens de plus en plus étroit entre rédaction technique et technologies numériques à travers les systèmes d'information embarqués (Muranko \& Drechsler, 2006), l'usage de la réalité virtuelle pour l'apprentissage de procédures (Ganier, Hoareau \& Devillers, 2013) ou encore l'usage d'assistants personnels électroniques ou de la réalité augmentée pour le guidage de techniciens de maintenance (Fiorentino et al., 2014 ; Ockerman, 2007 ; Toscano, 2000 ; Zafiharimalala, 2011).

- Des compétences dans la maîtrise du process de production des documents. En l'absence de la palette de compétences énumérées plus haut, le rédacteur technique doit posséder une bonne connaissance de l'ensemble du process de conception, afin d'être capable de mobiliser et de coordonner une équipe pluridisciplinaire (concepteur du produit, graphiste, traducteur, ergonome, etc.) autour de la conception du document.

\section{Conclusion}

Transmettre des connaissances procédurales par le biais de documents techniques constitue une activité complexe, mettant en jeu de multiples compétences. Dans le but de produire des documents adaptés aux utilisateurs et à leurs besoins, ces compétences gagneraient à être mieux développées via des cursus de formation structurés, encore trop peu développés en France. En s'appuyant sur les travaux de recherche réalisés jusqu'à présent, il est d'ores et déjà possible de délivrer des connaissances aux rédacteurs techniques sur la façon dont les individus interagissent avec les documents procéduraux (en s'appuyant sur les modèles existants et sur les résultats d'études empiriques) ou sur les stratégies de rédaction les plus efficaces. Il est également possible de les former à l'utilisation d'outils ou de méthodes destinés à évaluer la qualité ou l'efficacité de ces documents particuliers. Pour mieux travailler sur ces perspectives de formation des 
rédacteurs techniques, il est toutefois nécessaire d'initier ou d'approfondir certains travaux de recherches portant sur les questions suivantes : Comment développer des compétences liées à l'identification des besoins d'information des utilisateurs ? Comment faciliter la formalisation des connaissances d'un expert ? Comment évaluer les effets de différents formats de présentation sur le comportement des utilisateurs ? Comment faciliter l'acquisition de connaissances procédurales? Par ailleurs, le développement de l'usage des documents numériques et le développement de technologies comme la réalité virtuelle ou la réalité augmentée peuvent avoir de profondes conséquences sur la façon d'envisager la documentation technique et sur le métier de rédacteur technique. Ces mutations nécessitent d'approfondir les recherches sur les facteurs de présentation de l'information liés spécifiquement aux usages du numérique (animations graphiques, vidéo, etc.) et à la réalité virtuelle ou augmentée. Imaginer les possibilités offertes et savoir comment exploiter ces technologies constituent de nouveaux défis pour les rédacteurs techniques de demain.

\section{BIBLIOGRAPHIE}

BROADBENT, D. E. (1977). « Language and Ergonomics ». Applied Ergonomics 8 (1), p.15-18.

CHAPANIS, A. (1965). « Words, Words, Words ». Human Factors 7, p. 1-17.

CHAPANIS, A. (1988). « “Words, Words, Words" revisited ». International Reviews of Ergonomics 2, p. $1-30$.

CLERC, I. \& BEAUDET, C. (2002). « Pour un enseignement de la rédaction professionnelle ou de la rédaction technique?». Technostyle 17 (3), p. 27-44.

Coppola, N. W. (2011). « Professionalization of Technical Communication: Zeitgeist for our age » (Introduction to special issue, Part 1) ». Technical Communication 58 (4), p. 277-284.

FAYOL, M. (2002). « Les documents techniques: bilan et perspectives ». Psychologie Française 47 (1), p. 9-18.

FELKER, D. B. (1980). Document Design: A Review of the Relevant Research. Washington, DC: American Institutes for Research Technical Report 75002-4/80.

Fiorentino, M., UVA, A. E., GATTUllo, M., Debernardis, S. \& MonNo, G. (2014). « Augmented Reality on Large Screen for Interactive Maintenance Instructions ». Computers in Industry 65 (2), p. 270-278.

FLACKE, M.-L. (2005). « Technical Communication in France », in : J. Hennig \& M. Tjarks-Sobhani (eds.), Technical Communication International - Today and in the future. Lübeck: Schmidt-Römhild, p. 36-50.

GANIER, F. (2002a). «Évaluer l'efficacité des documents techniques procéduraux : un panorama des méthodes ». Le Travail humain 65 (1), p. 1-27.

GANIER, F. (2002b). «L'analyse des fonctionnements cognitifs : un support à l'amélioration de la conception des documents procéduraux ». Psychologie Française 47 (1), p. 53-64. 
GANIER, F. (2006). « La révision de textes procéduraux ». Langages 164, p. 71-85.

GANIER, F. (2008). «L'utilisation des documents procéduraux électroniques »In: Chevalier, A. \& Tricot, A. (éds.), Ergonomie cognitive des documents électroniques. Paris : PUF, p. 129-155.

GANIER, F. (2011). « L'ergonomie des modes d'emploi ». In: Dinet, J. \& Bastien, C. (éds), L'ergonomie des objets et environnements physiques et numériques. Paris : Hermès Sciences/Lavoisier, p. 65-84.

GANIER, F. (2012). « Cognitive Models of Understanding Procedural Instructions ». In: Rothkegel, A. \& Ruda, S. (eds), Communication on and via technology. Berlin: Mouton/ de Gruyter, p. 39-43.

GANIER, F. (2013). Comprendre la documentation technique. Paris : PUF.

GANIER, F. \& BARCENILlA, J. (2007). « Considering Users and Their Uses of Procedural Texts : a Prerequisite for the Design of Appropriate Documents ». In: Alamargot, D., Terrier, P. \& Cellier, J.M. (eds), Improving the Production and Understanding of Written Documents in the Workplace. Amsterdam: Elsevier, p. 49-66.

GANIER, F., GOMBERT, J. E. \& FAYOL, M. (2000). « Effets du format de présentation des instructions sur l'apprentissage de procédures à l'aide de documents techniques ». Le Travail humain 63 (2), p. 121-152.

GANier, F., HOAREAU, C. \& DEVILLERS, F. (2013). «Évaluation des performances et de la charge de travail induits par l'apprentissage de procédures de maintenance en environnement virtuel ». Le Travail humain 76 (4), p. 335-363.

GANIER, F. \& PÉTILLON, S. (2011). « Réviser un texte procédural en manipulant un dispositif : implications sur l'élaboration de différents niveaux de représentation et sur la qualité du texte produit ». Le Travail humain 74 (3), p. 225-251.

HAYES, J. R. \& FLOWER, L. (1980). « Identifying the Organization of Writing Processes ». In: Gregg, L. W. \& Steinberg, E. R. (éds.), Cognitive Processes in Writing. Hillsdale, NJ: Lawrence Erlbaum Associates, p. 3-30.

HEURLEY, L. \& GANIER, F. (2002). « La production de textes techniques écrits ». In: Fayol, M. (éd.), Traité des Sciences Cognitives : Production du langage. Paris : Hermès/Lavoisier, p. 229-249.

HEURLEY, L. \& GANIER, F. (2006). «L'utilisation des textes procéduraux : lecture, compréhension et exécution d'instructions écrites ». Intellectica 44, 2, p. 45-62.

JONES, S. (1968). « Instructions, Self Instructions and Performance ». Quarterly Journal of Experimental Psychology 20, p. 74-79.

JONES, S. (1966). « Decoding a Descriptive Instruction ». British Journal of Psychology 57, p. 405-411. KERN, R. P. (1985). « Modeling Users and Their Use of Technical Manuals ». In: Duffy, T. M. \& Waller, R.(eds), Designing Usable Texts. London: Academic Press, p. 341, 375.

LOPINTO, L. (1984). « Designing and Writing Operating Manuals ». IEEE Transactions on Professional Communication 27, p. 29-31.

MURANKO, B. \& DRECHSLER, R. (2006). « Technical Documentation of Software and Hardware in Embedded Systems ». In: Very Large Scale Integration, IFIP International Conference on, IEEE, $\mathrm{p}$. 261-266.

MYKITYSHYN, A. L., FISK, A. D. \& ROGERS, W. A. (2002). « Learning to Use a Home Medical Device: Mediating Age-Related Differences with Training ». Human Factors 44 (3), p. 354-364. 
OCKERMAN, J. (2007). « Task-guidance Systems and Procedure Context: Enabling Procedures to Enhance Worker Performance ». In: Alamargot, D. Terrier, P. \& Cellier, J.-M. (eds), Improving the Production and Understanding of Written Documents in the Workplace. Amsterdam: Elsevier, p. 217-230.

RUSSElL, P., BossÉ-ANDRIEU, J. \& CAJOLET-LAGANière, H. (1995). « Technical Writing in French in Canada: Results of Two Surveys ». Technostyle 12 (2), p. 49-75.

SCHRIVER, K. A. (1989). « Evaluating Text Quality : The Continuum from Text-Focused to ReaderFocused Methods ». IEEE Transactions on Professional Communication 32, p. 238-255.

SREMEC, B. (1972). «Instructions, Mechanical Ability and Performance ». Applied Ergonomics 3 (2), p. 98-100.

SUlLIVAN, M. A. \& CHAPANIS, A. (1983). « Human Factoring a Text Editor Manual ». Behaviour \& Information Technology 2 (2), p. 113-125.

TORRANCE, M., ALAMARGot, D., CASTELlo, M., GANiER, F., KRUSE, O., MANGEN, A., TOLCHINSKi, L. \& VAN WAES, L. (eds). (2012). Learning to Write Effectively: Current Trends in European Research. London: Emerald.

TOSCANO, L. (2000). « Electronic Technical Manual Development ». IEEE Aerospace and Electronic Systems Magazine 15 (8), p. 22-24.

VAlentin, A. \& LuCONGSANG, R. (1987). L'ergonomie des logiciels. Montrouge : Éditions de l'Anact. VANHUlle, S. (2000). La langue française à l'ouvrage. Paris : Duculot.

VeYrac, H., CelLier, J.-M. \& BerTRAND, A. (1997). « Modèle de l'opérateur et modèle du prescripteur. Le cas des consignes de résolution de situations incidentelles pour les conducteurs de trains ». Le Travail humain 60 (4), p. 387-407.

WESTENDORP, P. (1997). « Design Concepts of User Manuals ». In: Steehouder, M., Jansen, C., Poort, P. van der \& Verheijen R., Quality of Technical Documentation. USL\&C, Utrecht Studies in Language and Communication, p. 39-48.

WESTENDORP, P. (2000). « Reality, Theory and Design Aspects of Instructions for Use ». In: Communication at the Expert Forum Manual Design. International Institute for Information Design (IIID). Eskilstuna, Sweden: November 6-8.

WRIGHT, P. (1981a). Problems to be Solved when Creating Usable Documents ». In: Proceedings of IBM Symposium on Software and Information Usability, also available as HF077 from IBM Hursely, p. 53-103.

WRIGHT, P. (1981b). «Five Skills Technical Writers Need ». IEEE Transactions on Professionnal Communication 24 (1), p. 10-16.

WRIGHT, P. (1981c). « “The Instructions Clearly State...” Can't People Read ? ». Applied Ergonomics 12 (3), p. 131-141.

WRIGHT, P. (1987). « Writing Technical Information »,.Review of Research in Education 14, p. 327-385.

WRIGHT, P. (1988). «Issues of Content and Presentation in Document Design ». In: Helander, M. (ed.), Handbook of Human-Computer Interaction. Amsterdam: Elsevier, p. 629-652.

ZAFIHARIMALALA, H. (2011). Étude ergonomique pour la consultation sur écran de petite taille de la documentation de maintenance aéronautique. Thèse, Université de Toulouse Le Mirail. 


\section{NOTES}

1. Avec une cinquantaine de publications en une quarantaine d'années, Wright est à ce jour le seul auteur à avoir eu une production aussi importante dans ce domaine.

2. Dans cette partie, nous ne présenterons ni le process de conception de documents procéduraux en équipe, ni les processus cognitifs engagés par un rédacteur isolé, ces aspects ayant déjà été traités par Heurley et Ganier (2002).

\section{RÉSUMÉS}

Qu'il s'agisse de recettes de cuisine, d'aides en ligne ou encore de notices d'utilisation, les documents techniques procéduraux se distinguent d'autres types d'écrits par leur visée pragmatique: il s'agit de communiquer des procédures de façon efficace. Toutefois, ces documents, omniprésents dans la vie courante, échouent parfois dans la réalisation de cet objectif pour des raisons qui peuvent être multiples. Depuis plus de quarante ans, des études sont réalisées en psychologie cognitive et en ergonomie pour tenter d'identifier les facteurs impliqués dans l'utilisation de tels documents et pour proposer des solutions permettant d'en améliorer la qualité et l'efficacité. Ces études sont centrées en majeure partie sur l'utilisation et la compréhension de ces documents par le destinataire et accordent peu de place au rôle joué par le rédacteur technique, à son fonctionnement cognitif, au processus de rédaction, ou encore aux contraintes et aux difficultés rencontrées dans l'élaboration de tels documents. L'objectif de cet article est de s'appuyer sur les apports des travaux réalisés en psychologie cognitive et en ergonomie pour aider les rédacteurs techniques à améliorer l'efficacité des documents procéduraux. Préalablement à la présentation des travaux centrés sur le traitement des documents procéduraux par les utilisateurs, et destinés à identifier les facteurs cognitifs et ergonomiques pertinents à prendre en compte pour la conception de ces documents, il s'agira de déterminer les sources de difficultés auxquelles se trouvent confrontés les rédacteurs techniques. L'ensemble sera complété par une présentation des apports des recherches sur la conception et la rédaction de documents techniques, d'une part à travers une présentation des modèles du process de conception de documents techniques, et d'autre part à travers une présentation des compétences que doivent acquérir les rédacteurs techniques pour une communication efficace des procédures.

\section{INDEX}

Mots-clés : compréhension d'instructions, documents techniques, ergonomie de documents, procédures, rédaction technique 


\section{AUTEUR}

\section{FRANCK GANIER}

Université de Bretagne Occidentale, Université de Bretagne Sud, Telecom Bretagne, ENSTA Bretagne, École nationale d'ingénieurs de Brest, Centre national de la recherches scientifique, Lab-STICC, UMR 6285, F-29238, France 